\section{AN APPROACH TO THE BIOSYNTHESIS OF MACROLIDE ANTIBIOTIC PLATENOMYCIN}

Sir:

Basic 16-membered macrolide antibiotic platenomycins* produced by Streptomyces platensis subsp. malvinus MCRL 0388 ${ }^{11}$ were composed of two major components namely $A_{1}$ and $B_{1}$ and eleven other minor components ${ }^{2,3,4,5,6)}$. In this communication, we wish to describe the properties and structures of the five biogenetic intermediates of platenomycin obtained by blocked mutants together with their bioconversion to platenomycins $A_{1}, B_{1}$ and $C_{2}$.

Our intent was to pick blocked mutants of $S$. platensis subsp. malvinus which might produce biogenetic intermediates of platenomycin (PLM). Applying the procedure described by DeLIC et al., ${ }^{71}$ twenty-four non-platenomycin-producing strains obtained by NTG (N-methyl- $\mathrm{N}^{\prime}$-nitro$\mathrm{N}$-nitrosoguanidine) and/or UV treatment of a platenomycin-producing strain were tested for cosynthesis ability. These mutants were classified into 8 groups, and the complementation patterns between each group were as shown in Table 1. Data in Table 1 suggested that mutants of groups A, B, C and E were blocked on the main pathway of platenomycin biosynthesis, and it was thought that the strains of groups A and $\mathbf{B}$ which acted only as a secretor** might a producer of valuable biogeneticintermediates of platenomycin.

From cultured broth of the blocked mutants belonging to group $\mathrm{A}$ (strain $\mathrm{N}-90, \mathrm{~N}-33$ and U-253), 3-O-propionyl-5-O-mycaminosyl-platenolide I(III), II (IV) and demycarosyl-platenomycin (V) were isolated, and from that of another blocked mutants belonging to group $B$ (strain U-92 and N-22), platenolide I(I) and II(II) which are closely related to the platenomycin aglycone were isolated as major products.

The properties of these five products were as follows: Platenolide I (PL-I) (I), $\mathrm{C}_{20} \mathrm{H}_{32} \mathrm{O}_{6}$; m.p. $58 \sim 60^{\circ} \mathrm{C} ;[\alpha]_{D}^{25}+53^{\circ}\left(c 1, \mathrm{CHCl}_{3}\right) ; \lambda \max$ (EtOH) $279.5 \mathrm{~nm}(\log \varepsilon 4.32$ ); $\nu \max$ (nujol) $3470(\mathrm{OH}), 1725$ (lactone and ester), 1680 (ketone), 1630 and $1590 \mathrm{~cm}^{-1}$ (double bond); NMR (100 $\left.\mathrm{MHz}, \mathrm{CDCl}_{3}\right) \delta 0.90\left(3 \mathrm{H}, \mathrm{t},-\mathrm{CH}_{2}-\mathrm{CH}_{3}\right), 1.15 \sim$ $1.4\left(6 \mathrm{H}, 2 \times \mathrm{C}-\mathrm{CH}_{3}\right), 3.58\left(3 \mathrm{H}, \mathrm{s},-\mathrm{OCH}_{3}\right), 3.74$ $(1 \mathrm{H}, \mathrm{d}, \mathrm{J}=10.5 \mathrm{~Hz}, \mathrm{H}-3), 4.05(1 \mathrm{H}, \mathrm{d}, \mathrm{J}=9.3$ $\mathrm{Hz}, \mathrm{H}-5), 6.4 \sim 6.8(3 \mathrm{H}, \mathrm{m}$, olefinic), $7.25(1 \mathrm{H}$, $\mathrm{m}$, olefinic). I afforded diacetate by the usual acetylation procedure. The mass spectrum of diacetyl I showed $m / e \quad 452\left(\mathrm{M}^{+}\right), 392\left(\mathrm{M}^{+}-\right.$ $\left.\mathrm{CH}_{3} \mathrm{COOH}\right)$ and $333\left(\mathrm{M}^{+}-\mathrm{CH}_{3} \mathrm{COOH}-\right.$ $\mathrm{CH}_{3} \mathrm{COO} \cdot$ ).

Platenolide II (PL-II) (II), $\mathrm{C}_{20} \mathrm{H}_{34} \mathrm{O}_{6}$; m.p. $59 \sim 61^{\circ} \mathrm{C} ;[\alpha]_{\mathbb{D}}^{25}+12^{\circ}\left(c 1, \mathrm{CHCl}_{3}\right) ; \lambda \max (\mathrm{EtOH})$ $232 \mathrm{~nm}$ ( $\log \varepsilon 4.46$ ); $\nu \max$ (nujol) $3450(\mathrm{OH})$, $1710 \sim 1740$ (lactone and ester), 1660 and 1620 $\mathrm{cm}^{-1}$ (double bond); NMR (100 MHz, $\mathrm{CDCl}_{3}$ ) $\delta 0.95\left(3 \mathrm{H}, \mathrm{t},-\mathrm{CH}_{2}-\mathrm{CH}_{3}\right), 1.00 \sim 1.30(6 \mathrm{H}, 2 \times$ $\left.\mathrm{C}-\mathrm{CH}_{3}\right), 3.56\left(3 \mathrm{H}, \mathrm{s},-\mathrm{OCH}_{3}\right), 3.78(1 \mathrm{H}, \mathrm{d}, \mathrm{J}=$ $10.5 \mathrm{~Hz}, \mathrm{H}-3), 4.08(1 \mathrm{H}, \mathrm{d}, \mathrm{J}=9 \mathrm{~Hz}, \mathrm{H}-5), 4.4$ $(1 \mathrm{H}, \mathrm{dd}, \mathrm{J}=9.6,4.5 \mathrm{~Hz}, \mathrm{H}-9), 5.64 \sim 6.50(4 \mathrm{H}$, $\mathrm{m}$, olefinic). II afforded triacetate, of which mass spectrum showed $m / e 496\left(\mathrm{M}^{+}\right), 436\left(\mathrm{M}^{+}-\right.$ $\left.\mathrm{CH}_{3} \mathrm{COOH}\right), \quad 376\left(\mathrm{M}^{+}-2 \times \mathrm{CH}_{3} \mathrm{COOH}\right), \quad 316$ $\left(\mathrm{M}^{+}-3 \times \mathrm{CH}_{3} \mathrm{COOH}\right)$.

3-O-Propionyl-5-O-mycaminosyl-platenolide I (PL-I-MC) (III), $\mathrm{C}_{31} \mathrm{H}_{51} \mathrm{NO}_{10}$; m.p. $110 \sim 113^{\circ} \mathrm{C}$; $[\alpha]_{\mathrm{D}}^{25}+54.6^{\circ}\left(c 0.4, \mathrm{CHCl}_{3}\right) ; \lambda \max (\mathrm{EtOH}) 279.5$ $\mathrm{nm}(\log \varepsilon 4.33) ; \nu \max$ (nujol) $3545(\mathrm{OH}), 1745$

Table 1. Cosynthesis between groups of blocked mutants of Streptomyces platensis subsp. malvinus*

\begin{tabular}{|c|c|c|}
\hline Group & (Strain No.) & A B CDEFG \\
\hline A & $(\mathrm{N}-90, \mathrm{~N}-33, \mathrm{U}-253)$ & - CDEF- \\
\hline B & $(\mathrm{U}-92, \mathrm{~N}-22)$ & $-\mathrm{CDE}--$ \\
\hline $\mathrm{C}$ & $\begin{array}{l}(\mathrm{N}-32, \mathrm{U}-21, \mathrm{~N}-1, \mathrm{~N}-4, \\
\mathrm{N}-5, \mathrm{~N}-29)\end{array}$ & $--\mathrm{EC}-$ \\
\hline D & $(\mathrm{N}-9, \mathrm{NU}-29)$ & $--\mathbf{D}-$ \\
\hline $\mathrm{E}$ & $(\mathrm{N}-11)$ & $-E-$ \\
\hline $\mathbf{F}$ & $(\mathrm{N}-17)$ & $-\cdots$ \\
\hline $\mathrm{G}$ & $(\mathrm{N}-3, \mathrm{~N}-15, \mathrm{~N}-36, \mathrm{~N}-37)$ & - \\
\hline \multicolumn{3}{|c|}{$\begin{array}{l}\text { Doubtful (NU-1, NU-5, N-19, } \\
\qquad \mathrm{N}-27, \mathrm{NU}-58)\end{array}$} \\
\hline \multicolumn{3}{|c|}{ Complementation pattern } \\
\hline & $\overline{\mathrm{E}} \quad \overline{\mathrm{C}} \quad \overline{\mathrm{F}}$ & $\mathrm{A}$ \\
\hline & D & \\
\hline
\end{tabular}

* -: Indicated no cosynthesis; Cosynthesis was observed on the lettered position, when an inhibition zone appeared on the site of the group described.

* These antibiotics were previously reported as antibiotic YL-704.

** A secretor is a blocked mutant which can not produce an antibiotic substance by itself, but can produce a biogenetic intermediate. 
(lactone and ester), 1680 (ketone), 1635 and $1595 \mathrm{~cm}^{-1}$ (double bond); NMR (100 MHz, $\left.\mathrm{CDCl}_{3}\right) \delta \delta 0.74\left(3 \mathrm{H}, \mathrm{t},-\mathrm{CH}_{2}-\mathrm{CH}_{3}\right), \quad 1.0 \sim 1.4$ $\left(12 \mathrm{H}, 4 \times \mathrm{C}-\mathrm{CH}_{3}\right), 2.55\left(6 \mathrm{H}, \mathrm{s},-\overline{\mathrm{N}}\left(\mathrm{CH}_{3}\right)_{2}\right), 3.61$ $\left(3 \mathrm{H}, \mathrm{s},-\mathrm{OCH}_{3}\right), 3.89(1 \mathrm{H}, \mathrm{d}, \mathrm{J}=9.3 \mathrm{~Hz}, \mathrm{H}-5)$, $4.38\left(1 \mathrm{H}, \mathrm{d}, \mathrm{J}=6.9 \mathrm{~Hz}, \mathrm{H}-1^{\prime}\right), 5.90 \sim 6.40(3 \mathrm{H}$, $\mathrm{m}$, olefinic), $7.3(1 \mathrm{H}, \mathrm{m}$, olefinic). The mass spectrum of diacetyl III showed $m / e 681\left(\mathrm{M}^{+}\right)$, $621\left(\mathrm{M}^{+}-\mathrm{CH}_{3} \mathrm{COOH}\right), 407$ (aglycone ion: $\mathrm{AGL}^{+}$), $333\left(\mathrm{AGL}^{+}-\mathrm{C}_{2} \mathrm{H}_{5} \mathrm{COOH}\right), 258,198,156$ and 129. The latter four fragments were ascribed to a diacetyl mycaminose moiety in the molecule.

3-O-Propionyl-5-O-mycaminosyl-platenolide II (PL-II-MC) (IV), $\mathrm{C}_{31} \mathrm{H}_{53} \mathrm{NO}_{10} ;$ m.p. 114 $115^{\circ} \mathrm{C} ;[\alpha]_{\mathrm{D}}^{25}+31.5^{\circ}\left(\right.$ c $\left.0.4 \mathrm{CHCl}_{3}\right), \lambda \max (\mathrm{EtOH})$ $232 \mathrm{~nm}(\log \varepsilon 4.45) ; \nu \max$ (nujol) $3430(\mathrm{OH})$, 1735 (lactone and ester), 1665 and $1640 \mathrm{~cm}^{-1}$ (double bond); NMR (100 MHz, $\left.\mathrm{CDCl}_{3}\right) \delta 0.82$ $\left(3 \mathrm{H}, \mathrm{t},-\mathrm{CH}_{2}-\mathrm{CH}_{3}\right), 0.92 \sim 1.38\left(12 \mathrm{H}, 4 \times \mathrm{C}-\mathrm{CH}_{3}\right)$, $2.52\left(6 \mathrm{H}, \mathrm{s},-\widehat{\left.\mathrm{N}\left(\mathrm{CH}_{3}\right)_{2}\right)} 3.58\left(3 \mathrm{H},-\mathrm{OCH}_{3}\right), 3.90\right.$ $(1 \mathrm{H}, \mathrm{d}, \mathrm{J}=9.1 \mathrm{~Hz}, \mathrm{H}-5), 4.15(1 \mathrm{H}, \mathrm{dd}, \mathrm{J}=9.3$, $4.5 \mathrm{~Hz}, \mathrm{H}-9), 4.40\left(1 \mathrm{H}, \mathrm{d}, \mathrm{J}=6.6, \mathrm{H}-1^{\prime}\right), 5.5 \sim 6.8$ (4H, $\mathrm{m}$, olefinic). The mass spectrum of triacetyl IV showed $m / e 725\left(\mathrm{M}^{+}\right), 666\left(\mathrm{M}^{+}-\mathrm{CH}_{3} \mathrm{COO} \cdot\right)$, $606\left(\mathrm{M}^{+}-\mathrm{CH}_{3} \mathrm{COO} \cdot-\mathrm{CH}_{3} \mathrm{COOH}\right), 451\left(\mathrm{AGL}^{+}\right)$, $391\left(\mathrm{AGL}^{+}-\mathrm{CH}_{3} \mathrm{COOH}\right), 317\left(\mathrm{AGL}^{+}-\mathrm{CH}_{3}-\right.$ $\left.\mathrm{COOH}-\mathrm{C}_{2} \mathrm{H}_{5} \mathrm{COOH}\right), 258,198,156$ and 129.

Demycarosyl-platenomycin (DM-PLM) (V), $\mathrm{C}_{31} \mathrm{H}_{51} \mathrm{NO}_{11} ;$ m.p. $110 \sim 115^{\circ} \mathrm{C} ;[\alpha]_{\mathrm{D}}^{27}-8^{\circ}(\mathrm{c}$ $0.5, \mathrm{CHCl}_{3}$ ); $\lambda \max (\mathrm{EtOH}) 232 \mathrm{~nm}(\log \varepsilon 4.44)$; $v \max$ (nujol) $3420(\mathrm{OH}), 2715$ (-CHO), 1730
Chart 1. Biosynthetic pathway of platenomycins

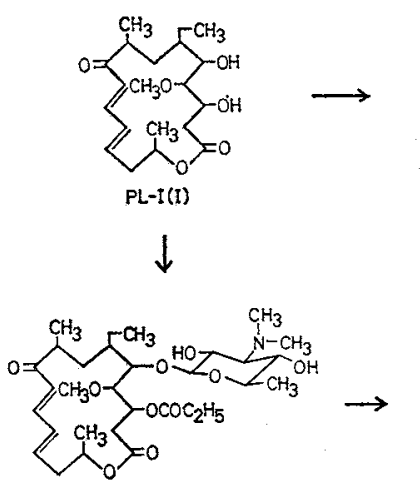

PL-I-MC(III)
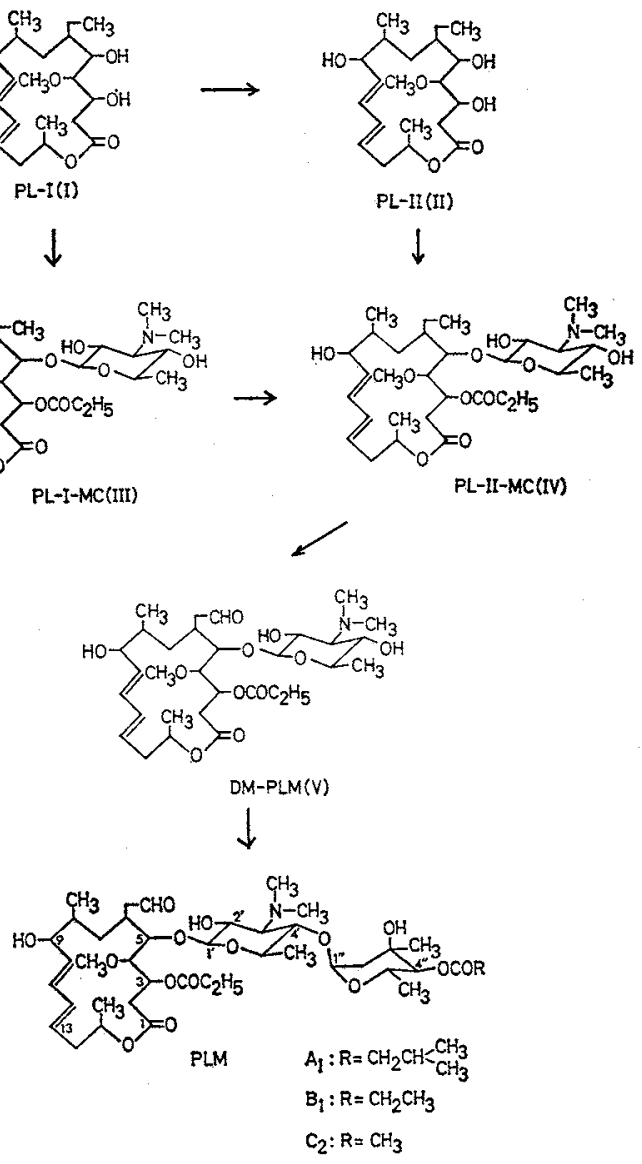

Table 2. The mutual bioconversion of five intermediates by washed mycelium of blocked mutants.

\begin{tabular}{|c|c|c|c|c|c|c|c|c|c|c|c|c|}
\hline \multirow[b]{2}{*}{ Group } & \multirow[b]{2}{*}{ Strain } & \multirow[b]{2}{*}{$\begin{array}{l}\text { Added } \\
\text { substrate }\end{array}$} & \multicolumn{8}{|c|}{$\begin{array}{l}\text { Ratio of substrate and bioconverted products } \\
\text { (mole } \%)\end{array}$} & \multirow[b]{2}{*}{ RS } & \multirow[b]{2}{*}{ BP } \\
\hline & & & PL-I & PL-II & $\begin{array}{l}\text { PL-I- } \\
\text { MC }\end{array}$ & $\begin{array}{l}\text { PL- } \\
\text { II- } \\
\text { MC }\end{array}$ & $\begin{array}{l}\text { DM- } \\
\text { PLM }\end{array}$ & $\begin{array}{c}\text { PLM- } \\
\mathrm{A}_{1}\end{array}$ & $\begin{array}{c}\mathrm{PLM}- \\
\mathbf{B}_{1}\end{array}$ & $\underset{\mathrm{C}_{2}}{\mathrm{PLM}}$ & & \\
\hline $\mathrm{C}$ & $\mathrm{N}-32$ & $\begin{array}{l}\text { PL-I } \\
\text { PL-II } \\
\text { PL-I-MC } \\
\text { PL-II-MC } \\
\text { DM-PLM }\end{array}$ & 0 & $\begin{array}{l}22.6 \\
31.7\end{array}$ & 0 & $\begin{array}{l}12.5 \\
34.9 \\
88.0 \\
72.9\end{array}$ & $\begin{array}{l}17.4 \\
12.0 \\
27.1 \\
59.2 \\
\end{array}$ & $\begin{array}{r}11.0 \\
9.4 \\
\\
18.6 \\
\end{array}$ & $\begin{array}{l}27.6 \\
18.3 \\
20.1 \\
\end{array}$ & $\begin{array}{l}8.9 \\
5.7 \\
2.1\end{array}$ & $\begin{array}{c}0 \\
28.8 \\
0 \\
65.8 \\
47.1\end{array}$ & $\begin{array}{r}81.3 \\
87.0 \\
100.8 \\
71.2 \\
61.1\end{array}$ \\
\hline E & $\mathrm{N}-11$ & $\begin{array}{l}\text { PL-I } \\
\text { PL-II } \\
\text { PL-I-MC } \\
\text { PL-II-MC } \\
\text { DM-PLM }\end{array}$ & 77.1 & $\begin{array}{l}15.0 \\
95.5\end{array}$ & 97.5 & 85.6 & 81.0 & $\begin{array}{l}5.1 \\
7.1 \\
\end{array}$ & $\begin{array}{l}4.5 \\
2.5 \\
7.3 \\
7.2 \\
\end{array}$ & $\begin{array}{l}2.0 \\
4.7\end{array}$ & $\begin{array}{l}67.6 \\
94.1 \\
95.9 \\
84.1 \\
84.1 \\
\end{array}$ & $\begin{array}{r}69.9 \\
80.0 \\
76.3 \\
89.8 \\
105.1 \\
\end{array}$ \\
\hline $\mathrm{F}$ & $\mathrm{N}-17$ & $\begin{array}{l}\text { PL-I } \\
\text { PL-II } \\
\text { PL-I-MC } \\
\text { PL-II-MC } \\
\text { DM-PLM }\end{array}$ & $\begin{array}{l}46.7 \\
23.0\end{array}$ & $\begin{array}{r}53.3 \\
100.0 \\
40.6\end{array}$ & 0 & $\begin{array}{l}24.6 \\
71.0\end{array}$ & $\begin{array}{r}9.0 \\
29.0 \\
77.5\end{array}$ & 7.2 & $\begin{array}{r}2.8 \\
15.3\end{array}$ & & $\begin{array}{c}46.0 \\
82.8 \\
0 \\
60.9 \\
75.9\end{array}$ & $\begin{array}{r}104.1 \\
0 \\
105.3 \\
63.6 \\
101.7 \\
\end{array}$ \\
\hline
\end{tabular}

MC: Mycaminosyl glycoside

RS: Recovery of substrate (mole \%), BP: Yield of bioconverted products [mole \%, (Total bioconverted products/(Added substrate-Recovered substrate)) $\times 100$ ].

Medium: $0.25 \%$ glucose-physiological saline solution. Cell concentration: $0.4 \mathrm{~g}$ wet weight/ ml. Added substrate concentration: $200 \sim 300 \mu \mathrm{M}$. Incubation condition: $28^{\circ} \mathrm{C}, 200$ r.p.m., 5 hours 
(lactone and ester), 1650 and $1630 \mathrm{~cm}^{-1}$ (double bond); NMR (100 MHz, $\left.\mathrm{CDCl}_{3}\right) \delta 1.02(3 \mathrm{H}, \mathrm{d}$, $\left.\mathrm{J}=6.9 \mathrm{~Hz}, \mathrm{C}-\mathrm{CH}_{3}\right), 1.14 \sim 1.40\left(9 \mathrm{H}, 3 \times \mathrm{C}-\mathrm{CH}_{3}\right)$, $2.55\left(6 \mathrm{H}, \mathrm{s},-\mathrm{N}\left(\mathrm{CH}_{3}\right)_{2}\right), 3.58\left(3 \mathrm{H}, \mathrm{s},-\mathrm{OCH}_{3}\right)$, $3.94(1 \mathrm{H}, \mathrm{d}, \mathrm{J}=9.3 \mathrm{~Hz}, \mathrm{H}-5), 4.13(1 \mathrm{H}, \mathrm{dd}, \mathrm{J}=$ 9.1, $4.5 \mathrm{~Hz}, \mathrm{H}-9), 4.46\left(1 \mathrm{H}, \mathrm{d}, \mathrm{J}=7.2 \mathrm{~Hz}, \mathrm{H}-1^{\prime}\right)$, $5.5 \sim 6.9(4 \mathrm{H}, \mathrm{m}$, olefinic), $9.59(1 \mathrm{H}, \mathrm{s},-\mathrm{CHO})$. The mass spectrum of triacetyl $\mathrm{V}$ showed $\mathrm{m} / \mathrm{e}$ $739\left(\mathrm{M}^{+}\right), 711\left(\mathrm{M}^{+}-\mathrm{H}_{2} \mathrm{O}\right), 465\left(\mathrm{AGL}^{+}\right), 437$ $\left(\mathrm{M}^{+}-\mathrm{CO}\right), 405\left(\mathrm{AGL}^{+}-\mathrm{CH}_{3} \mathrm{COOH}\right), 331\left(\mathrm{AGL}^{+}-\right.$ $\left.\mathrm{CH}_{3} \mathrm{COOH}-\mathrm{C}_{2} \mathrm{H}_{5} \mathrm{COOH}\right), 258,198,156$ and 129. $V$ was identified with demycarosyl-YL-704 $\mathrm{A}_{1}-\mathrm{I}^{5)}$ which was obtained by the hydrolysis of platenomycin $A_{1}$.

Comparing the above data with those of platenomycin $\mathrm{A}_{1}{ }^{2,5\rangle}$ and $\mathrm{W}_{1}{ }^{3,6\}}$ the UV absorption maximum at $232 \mathrm{~nm}$ indicated the presence of the $\alpha, \beta, \gamma, \delta$-dienol chromophore as platenomycin $A_{1}$ and the maximum at $280 \mathrm{~nm}$ showed the presence of the $\alpha, \beta, \gamma, \delta$-dienone chromophore like platenomycin $W_{1}$. From the NMR spectra, $\mathrm{V}$ contained an aldehyde function at a $\mathrm{C}-18$ position, while other four products, I, II, III and IV, contained a methyl residue instead of an aldehyde function on the aglycone skeleton. This was also supported by IR spectra. Moreover, it was clearly observed from their mass spectra that these five products and their acetyl derivatives did not contain the fragments due to an acyl-mycarose moiety, and, further I and II did not show any fragments relating to a mycaminose moiety. From the above physicochemical data, the structures of the five products $(\mathbf{I} \sim \mathrm{V})$ produced by the blocked mutants of groups A and B were elucidated as depicted in Chart 1.

As shown in Table 2, the above five products were readily converted to platenomycins $A_{1}$, $B_{1}$ and $C_{2}$ by washed mycelium of the blocked mutants belonging to groups $\mathrm{C}$ and $\mathrm{E}$. However, in the case of a blocked mutant belonging to group F (strain N-17), I and II were not converted to III, IV, $\mathrm{V}$ and platenomycins. Biosynthetic pathway involving such products were summarized in Chart 1 . These bioconversion behaviors supported the structure of the intermediates, and also were not incompatible with the above-mentioned cosynthetic data between blocked mutant groups.

Consequently, it is suggested that these five products are intermediates of platenomycin biogenesis. Bioconversion of PL-I to PL-I-MC was not observed by mutants of groups $\mathrm{C}$ and $\mathrm{E}$, but this conversion was observed by blocked mutants of group A.

The details of these studies will be published elsewhere in the near future.

\section{Acknowledgement}

The authors wish to aknowledge Dr. T. OKUDA, manager of this laboratory for his encouragement, and Dr. K. KoterA and his collaborators of Analytical Center of this company for the instrumental and elemental analyses.

Tamotsu Furumai
Yoshiaki SeKI
Katsuo Takeda
Akio Kinumaki
Makoto SuzUKI
Microbial Chemistry Research Laboratory
Tanabe Seiyaku Co., Ltd.
Toda, Saitama, Japan

(Received August 10, 1973)

\section{References}

1) Furumai, T.; Y.Shimizu, K.Takeda, N. MatsuZaWA, K. Tani \& T. OKuda: Studies on the macrolide antibiotic YL-704 complex. I. Taxonomy and production of the producing strain. J. Antibiotics (in press)

2) SuzukI, M.; I. Takamori, A. Kinumaki, Y. Sugawara \& T. OKUdA: The structures of antibiotic YL-704 A and B. Tetrahedron Letters 1971-5: 435 438, 1971

3) Suzuki, M.; I, Takamori, A. Kinumaki, Y. SUGAWARA \& T. OKUDA: The structures of antibiotics $Y_{L}-704 C_{1}, C_{2}$ and $W_{1}$. J. Antibiotics 24: 904 906, 1971

4) Kinumaki, A.; I. Takamori, Y. Sugawara, N. Nagahama, M. Suzuki, Y. Egawa, M. SakuRAZAWA \& T. OKUDA: Studies on the macrolide antibiotics YL-704 complex. II. Isolation and physicochemical properties of $\mathrm{YL}$ 704 components. J. Antibiotics (in press)

5) Kingmaki, A.; I. Takamori, Y. Sugawara, M. SuzukI \& T. OKUDA: Studies on the macrolide antibiotic YL-704 complex. III. The structures of antibiotic YL-704 $A_{1}$ and $B_{1}$. J. Antibiotics (in press)

6) Kinumaki, A.; I. Takamori, Y. Sugawara, Y. SeKI, M. SuzuKI \& T. OKuda: Studies on the macrolide antibiotic YL-704 complex. IV. The structures of minor components. J. Antibiotics (in press)

7) Delic, V.; J. Pigac \& G. Sermonti: Detection and study of cosynthesis of tetracycline antibiotics by an agar method. J. Gen. Microbiol. 55: $103 \sim 108,1969$ 